\title{
Vibrotactile difference thresholds for intensity and the effect of a masking stimulus*
}

\author{
JAMES C. CRAIG \\ Indiana University, Bloomington, Indiana 47401
}

\begin{abstract}
Vibrotactile difference thresholds for intensity were measured at several intensity levels of a test stimulus in the absence of a masking vibration and in the presence of three different amplitudes of a masking vibration. The test stimulus was a $160-\mathrm{Hz}$ vibration delivered to the right index finger. The masking stimulus was a $160-\mathrm{Hz}$ vibration delivered to the right little finger. For the same amplitudes of the test stimulus, $\Delta I$ varied as a direct function of the amplitude of the masking vibration. The smallest $\Delta$ Is resulted from measurements made in the absence of the masking stimulus. The Weber fraction, $\Delta \mathrm{I} / \mathrm{I}$, was constant only for the more intense test stimuli in the absence of any masking stimuli. Independent of the presence or level of the masker, the Weber fraction for all stimuli approached approximately the same value, .25 , when the test stimuli were raised to 20 -dB sensation level. A model is proposed to account for the increase in the Weber fraction as a function of masker intensity and to predict masked thresholds.
\end{abstract}

In a recent publication, Craig (1972) examined the difference threshold (DL) for intensity for vibrotactile stimuli. The results showed that the presence of a masking stimulus raised the DL considerably, particularly when signals were less than $15 \mathrm{~dB}$ above their masked threshold. At intensity levels greater than $15 \mathrm{~dB}$ above threshold, the $\mathrm{DL}$ in the presence of a masking stimulus was similar to the DL in the absence of a masking stimulus. One aim of the present study was to measure DLs in the absence of a masking stimulus at intensity levels lower than those employed in the earlier study. If the DL increased as a function of decreasing intensity, it might be possible to fit the Weber functions obtained in the absence and in the presence of a masking stimulus with a single function.

Craig employed a masking stimulus which consisted of the output of a white-noise generator imposed on the same vibrator through which the signal was presented. Several recent attempts to develop cutaneous communication systems have employed a number of vibrators placed at various loci on the body. If intensity differences among vibrators were to be used as a cue in such systems, it would be important to know the effect of a masking vibrator located at a site different from that of the test vibrator. Moreover, cutaneous communication systems typically use sinusoidal stimuli of the same frequency. A second aim of the present study was to extend Craig's results to include the effect of a masking stimulus of the same frequency as the test stimulus, but located at a different site on the skin.

A third aim was to formulate a model for intensity discrimination which would account for the increase in the size of the DL in the presence of a masking vibration and would also predict changes in detection in the presence of a masking vibration.

*This study was supported by Grant NS-09783 from the National Institutes of Health, U.S. Department of Health, Education and Welfare.

\section{METHOD}

\section{Observers}

The Os were three paid undergraduates. Two of the Os were female, one was male. All Os received several hours of practice before data collection was begun.

\section{Apparatus}

The timing and presentation of the stimuli were controlled by Grason-Stadler Series 1200 modules. The test stimuil were generated by the output of a Hewlett-Packard $3300 \mathrm{~A}$ oscillator which was delivered to each of two Grason-Stadler 1287 electronic switches. The outputs of the electronic switches were amplified by Krohn-Hite DCA-10 amplifiers, the outputs of which were attenuated and led to an impedance matching transformer. The output of the matching transformer was fed to a Goodmans V-47 vibrator. The vibrator rested on a balance to insure a constant $20 \mathrm{~g}$ of static force against the O's finger. The vibrator was fitted with a circular contactor, $6 \mathrm{~mm}$ in diam, which protruded through a fixed surround, $8 \mathrm{~mm}$ in diam. A Grason-Stadler 1240 probability generator randomly determined which of the two electronic switches would be turned on in the first observation interval and which in the second observation interval. When the DL was measured in the presence of a masking stimulus, a second vibrator, identical to the first, was turned on by a third electronic switch. An MB vibration meter, M-7, with a Type 115 pickup was used to measure the amplitude of vibration as a function of the voltage imposed on the coil of the vibrator. A plot of peak-to-peak amplitude vs voltage was linear for the range of voltages examined. Extrapolation of the function was necessary in order to arrive at an estimate of some of the smaller amplitudes which did not register on the vibration meter.

\section{Procedure}

Measurements were made of three different thresholds, the absolute threshold for the test stimulus (quiet threshold), the threshold for the test stimulus in the presence of the masking stimulus (masked threshold), and the DL for the test stimulus. All thresholds were measured using a two-interval, temporal forced-choice procedure with a block up-and-down method (Campbell, 1963). The method concentrates observations in the region near threshold and was used to determine the intensity 


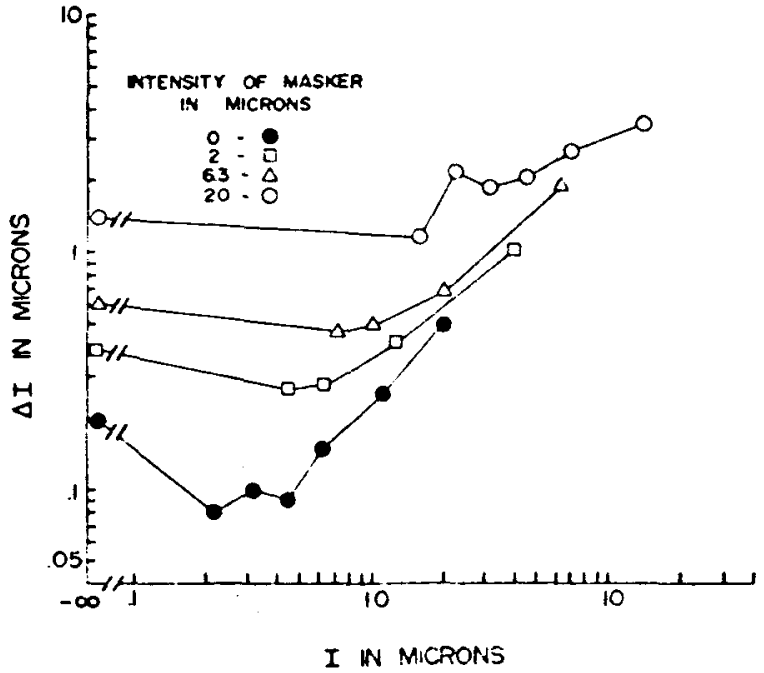

Fig. 1. $\Delta I$ in microns peak-to-peak as a function of $I$ in microns peak-to-peak. The parameter is the intensity of a masking stimulus in microns peak-to-peak. The points at $-\infty$ represent absolute and masked threshold measurements.

level at which the $\mathrm{O}$ responded correctly $75 \%$ of the time. When DLs were measured, the intensity of the stimuli was changed in .3-dB steps. When either absolute or masked thresholds were measured, the step size was $1 \mathrm{~dB}$. Approximately 100 trials were run to determine each threshold. See Craig (1972) for additional information concerning this threshold procedure.

The sequence of events on every trial was as follows: a 1-sec warning light, a $1-\mathrm{sec}$ light indicating the first observation interval, and another $1-\mathrm{sec}$ light indicating the second observation interval. An answer period followed the observation intervals during which the $O$ pressed one of two buttons which corresponded to the observation intervals. Feedback was given to indicate whether the $O$ was correct or not. When the quiet threshold was being measured, the $O$ received a stimulus in one of the two observation intervals. He pressed the button corresponding to the interval in which he felt the stimulus was presented. When the masked threshold was being determined, the $O$ received the same masking stimulus in both observation intervals and the test stimulus in one of the intervals. He selected the interval in which he thought the test stimulus was presented. When the DL was measured, the $O$ received two test stimuli of different intensity, one in each interval. If the DL was measured in the presence of a masking stimulus, a masking stimulus was presented simultaneously with the test stimuli in each interval. The $O$ was instructed to indicate the interval which contained the more intense of the two test stimuli.

Both the test and the masking stimuli were bursts of $160-\mathrm{Hz}$ vibration with a rise time of $10 \mathrm{msec}$. The duration of both stimuli was $200 \mathrm{msec}$. The test stimulus was delivered to the right index finger, the masking stimulus was delivered to the right little finger. To measure DLs in the absence of a masking vibration, an experimental session consisted of determining the absolute threshold for the test stimulus, raising the intensity of the test stimulus to $1,4,7,10,15$, or $20 \mathrm{~dB} S \mathrm{~S}$ and measuring the $\mathrm{DL}$ at that intensity level. To measure the $\mathrm{DL}$ in the presence of a masking stimulus, an experimental session consisted of measuring the absolute threshold for the test stimulus in the presence of the masking stimulus, raising the intensity of the test stimulus to $1,4,7,10,15$, or $20 \mathrm{~dB}$ above the masked threshold, and measuring the $\mathrm{DL}$ at that intensity. The difference in the amplitude of vibration required to detect the stimulus in the absence and in the presence of the masking stimulus was taken as the measure of the amount of masking. The masking stimulus was set at an amplitude of 20 microns peak-to-peak, which is approximately $40 \mathrm{~dB}$ above absolute threshold. Some additional measurements were also made with masking stimuli of 6.3 microns peak-to-peak and 2.0 microns peak-to-peak, which are 10 and $20 \mathrm{~dB}$ less intense, respectively, than the first masking stimulus. Measurements made with the two less intense masking stimuli were made with the test stimulus raised to $1,4,10$, or $20 \mathrm{~dB}$ above masked threshold. These DL determinations were made after the measurements with the 20-micron masker. Additional measurements with the 20 -micron masker did not show any long-term change in the Os' sensitivity.

\section{RESULTS AND DISCUSSION}

The basic data are presented in Fig. 1, which shows $\Delta \mathrm{I}$ as a function of $I$ in $\log \cdot \log$ coordinates. The three Os showed similar results across conditions. The maximum difference between an individual O's mean absolute or masked threshold and the overall mean threshold was $2.5 \mathrm{~dB}$. For the DL measurements, the maximum difference between an individual O's mean DL and the overall mean $\mathrm{DL}$ exceeded $\mathrm{IdB}$ in only one instance, with most differences being less than $.5 \mathrm{~dB}$. Because of the similarity in performance, the Os' results have been combined. For the measurements in the absence of a masking stimulus, each point represents the mean of 21 observations (100 trials per observation). For the measurements in the presence of the 20-micron masking stimulus, each point represents the mean of 13 observations. Each point on the other two curves in the presence of the masking stimulus represents seven observations per point. The data points at minus infinity represent absolute and masked threshold measurements. The differences in amplitude between the data point at minus infinity with a zero-amplitude masker and the data points with the 2.0-, 6.3-, and 20-micron maskers represent the amount of threshold shift produced by each of the maskers.

If $\Delta \mathrm{I} / \mathrm{I}$ were a constant, the resulting functions would be a straight line with a slope of unity. Plotting $\Delta l$ as a function of $I$ in the absence of a masking stimulus results in a linear function at higher intensities with a slope close to unity. The functions obtained in the presence of a masking stimulus show slopes somewhat less than unity. The general trend is: the greater the intensity of the masking stimulus, the lower the slope.

The results from Fig. 1 were replotted in Fig. 2 in order to show that $\Delta I / I$ does not remain constant as the intensity of the masking stimulus is increased. A second vertical scale, in decibels, shows the difference in at tenuator settings between $\mathrm{I}$ and $\mathrm{I}+\Delta \mathrm{I}$, i.e., $20 \log [(\mathrm{I}+\Delta \mathrm{I}) / \mathrm{I}]$. It is clear that the presence of a masker increases the Weber fraction, with the most intense masker producing the largest fraction. For example, for signals with an amplitude of approximately 2 microns, $\Delta \mathrm{I} / \mathrm{I}$ may be as small as .23 or as large as nearly 1.0 , depending upon the intensity of the masker. This result is similar to that obtained by Craig (1972).

The question remains whether or not when $\Delta \mathrm{I} / \mathrm{I}$ is plotted not as a function of absolute intensity but rather as a function of relative intensity, i.e, intensity level 
above threshold, the Weber functions are similar for masked and quiet data. To obtain such functions, the data from Fig. 2 were replotted in Fig. 3 to show $\Delta, I / I$ as a function of decibels either above quiet threshold, in the case of the zero masker condition, or above masked threshold, in the cases in which a masker was present.

Looking first at the Weber function in the quiet and comparing it to Craig's (1972) results makes it clear that, while $\Delta \mathrm{I} / \mathrm{I}$ increases somewhat at low intensities, the increase is not nearly large enough to fit the Weber function obtained in the presence of a masking stimulus. The same conclusion may be drawn from examining the curves in Fig. 3. When the data are plotted as a function of intensity above threshold, masked or quiet threshold as in Fig. 3, it is not possible to fit the data with a single curve.

\section{MODEL FOR DL AND MASKED THRESHOLD}

One of the major questions these data raise is: How can the increase in $\Delta \mathrm{I} / \mathrm{I}$ in the presence of a masking stimulus be accounted for? The proposed model attempts to fit the $\Delta \mathrm{I} / \mathrm{I}$ functions obtained in the presence of a masker to the $\Delta \mathrm{I} / \mathrm{I}$ function obtained in the absence of a masker and also to predict the masked threshold in the presence of the various maskers. The model attempts to demonstrate that, at a comparable intensity level above threshold, the same relative change in amplitude can be discriminated independently of the presence or level of the masker. The data presented as they are in Fig. 3 obviously do not support such a conclusion. However, it might be argued that plotting $\Delta \mathrm{I} / \mathrm{I}$ in the presence of a masker, where $\mathrm{I}$ is the intensity of the test signal, is not reasonable, because the $O$ is receiving, in addition to a test signal, a masker in both observation intervals. It might be assumed that a masker is adding some proportion of its amplitude to the test

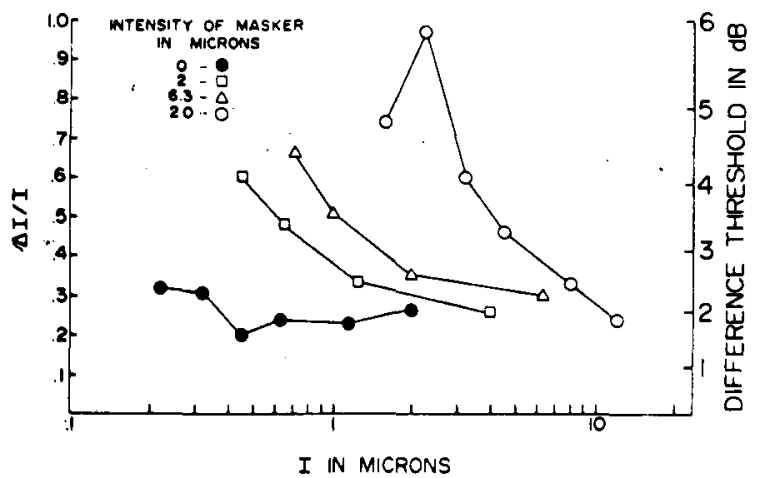

Fig. 2. Data from Fig. 1 replotted to show $\Delta I / I$ as a function of $I$ in microns peak-to-peak. The parameter is the intensity of a masking stimulus in microns peak-to-peak. A second vertical scale in decibels has been added to facilitate comparisons with earlier studies.

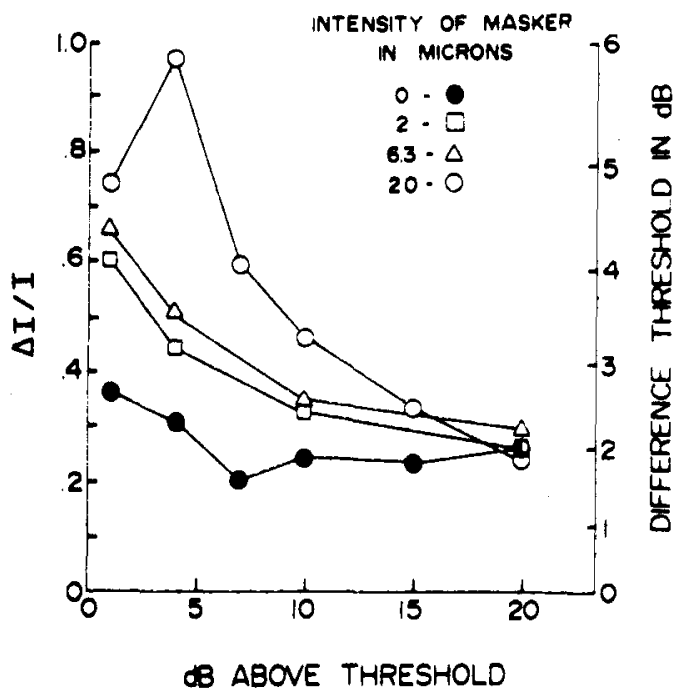

Fig. 3. Data from Fig. 2 replotted to show $\Delta I / I$ as a function of decibel sensation level. A second vertical scale in decibels has been added to facilitate comparisons with earlier studies.

site so that the $\mathrm{O}$ must discriminate between the intensity of the signal, $I_{s}$, plus some intensity contributed by the masker, $I_{m}$, and $I_{s}$ plus $I_{m}$ plus $\Delta I$. the increment added to the signal. Therefore, the result that should be plotted is $\Delta \mathrm{I} /\left(\mathrm{I}_{\mathrm{s}}+\mathrm{I}_{\mathrm{m}}\right)$.

To arrive at an estimate of $I_{m}$, the following equation may be solved for $I_{m}$ at each comparable intensity level above threshold for the 20-micron masker condition:

$$
\frac{\Delta \mathrm{I}}{\mathrm{I}_{\mathbf{s}}+\mathrm{I}_{\mathrm{m}}} \text { (with masker) }=\frac{\Delta \mathrm{I}}{\mathrm{I}} \text { (without masker). }
$$

The mean amplitude which must be added to each $\mathrm{I}_{\mathrm{S}}$ in the 20-micron condition in order to give the same $\Delta \mathrm{I} / \mathrm{I}$ as that obtained without the masker is 3.23 microns or $I_{m}=3.23$. It seems reasonable to assume that if 3.23 microns from the 20 -micron masker is contributing to the intensity discrimination at the test site, a similar proportion is being contributed by the less intense maskers. Assuming that the same proportion of the masker, $3.23 / 20$, is being contributed by the 2.0 and 6.3-micron maskers, the following equations may be solved for $I_{m}$ :

$$
\frac{3.23}{20}=\frac{\mathrm{I}_{\mathrm{m}}}{6.3} \text { and } \frac{3.23}{20}=\frac{\mathrm{I}_{\mathrm{m}}}{2.0}
$$

which yield values of $I_{m}=1.02$ microns for the 6.3 -micron masker and $\mathrm{I}_{\mathrm{m}}=0.32$ microns for the 2.0-micron masker. These values are similar to those which are obtained if average $I_{m}$ values are calculated in the same way as the 3.23 microns was found for the 20-micron masker condition, viz, 0.80 and 0.27 microns 


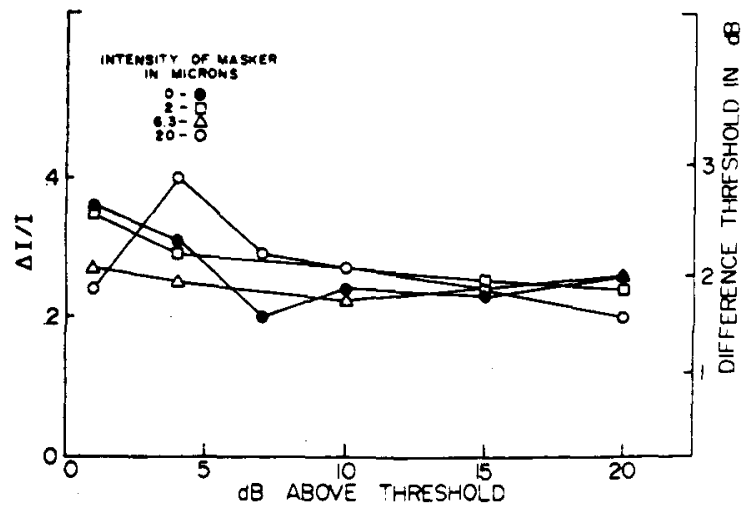

Fig. 4. Data from Fig. 3 replotted to show $\Delta I / I$ as a function of decibel sensation level with the constant amplitudes contributed by the masking stimuli added to I. A second vertical scale has been added to facilitate comparisons with earlier studies.

for the 6.3. and 2.0-micron masker conditions, respectively.

The values of $I_{m}$ of $3.23,1.02$, and 0.32 were added to Eq. 1 and $\Delta \mathrm{I} / \mathrm{I}$ was recalculated for each of the masker conditions. The resulting functions are shown in Fig. 4. A comparison of Fig. 4 with Fig. 3 shows that the curves with and without a masker present are much closer to being fitted by a single function in Fig. 4 than in Fig. 3. A similar model for auditory difference limens measured in background noise has been suggested by Sherrick (1959).

If a constant proportion of the masker is being added to the test stimulus, then it might be possible to use the same constants to predict performance in another psychophysical task, masked thresholds. Jeffress (1971) has pointed out that for auditory stimuli the quiet threshold, masked threshold, and the DL all involve similar tasks, an $O$ detecting some increase in energy. The quiet threshold is viewed as a DL task in which the $O$ is discriminating between one interval which contains some "noise" and a second interval which contains a similar amount of noise plus the signal. An estimate of the amplitude of this noise may be made from Fig. 3. In the absence of any masker, $\Delta \mathrm{I} / \mathrm{I}$ at $0 \mathrm{~dB}$ SL would be approximately 0.4 . In the case of quiet threshold measurements, $\Delta \mathrm{I}$ is the intensity of the signal, 0.2 microns, and $I$ is the intensity of the noise. If $\Delta I / I=$ 0.4 , then 0.5 microns is the calculated amplitude of the noise under the present conditions.

For the masked threshold measurements, it is assumed that the $O$ is discriminating one interval which contains noise plus some amplitude contributed by the masker from a second interval which contains the same noise plus the same amplitude contributed by the masker plus the signal. As in the DL measurements, it is also assumed that Os can detect the same relative change in amplitude with and without a masker present. At $0 \mathrm{~dB} \mathrm{SL}$, masked threshold, the equation for the 20-micron masker would be:

$$
\frac{\Delta \mathrm{I}}{0.5+3.23}=0.4
$$

where $\Delta \mathrm{I}$ is the amplitude of the signal required for $75 \%$ correct detection, 3.23 is the contribution of the 20-micron masker estimated from the DL measurements, and 0.5 is the calculated amplitude of the noise. Solving the above equation for $\Delta \mathrm{I}$ yields an estimate of the amplitude of the signal required to be detected in the presence of the 20-micron masker of 1.49 microns, which is close to the observed value of masked threshold of 1.41 microns. In a similar fashion, the signal amplitudes for the 6.3- and 2.0-micron masker conditions may be calculated. The calculated amplitudes are 0.61 and 0.33 microns, respectively, which also correspond closely to the observed values of 0.63 and 0.40 microns.

In calculating the masked threshold, it was necessary to assume the presence of noise, calculated to be 0.5 microns. If 0.5 microns is added to the $\mathrm{DL}$ measurements in the absence of a masker, $\Delta \mathrm{I} / \mathrm{I}$ became an increasing rather than a decreasing function of $I$. Because it seemed unlikely that $\Delta \mathrm{I} / \mathrm{I}$ would increase as a function of $\mathrm{I}$, the noise value was not added to the $\mathrm{DL}$ functions. This, of course, raises the question of whether or not it is reasonable to assume that noise is a factor in the masked and quiet threshold discriminations but is not a factor in the DL discriminations. The chief difference between the DL and the threshold measurements is that in the DL measurements a sinusoidal signal at the test site is present in both observation intervals. One explanation might be that the signal tends to modulate the noise, make it more regular, and allow the $\mathrm{O}$ to cancel, to some extent, the noise from the two observation intervals when the signal is presented in both observation intervals. Recordings from first-order cutaneous afferents has shown that there tends to be irregular firing at very low stimulus amplitudes, but at amplitudes corresponding to threshold for human detection, there is frequency-following of the stimulus input (Talbot, Darian-Smith, Komhuber, \& Montcastle, 1968). Admittedly, this is a very tentative explanation. However, it is the case that if some kind of noise is not assumed to be present in the threshold discrimination, predictions of the masked threshold are not possible from the DL measurements. Clearly, additional measurements of the masked and quiet threshold and the DL are necessary, perhaps on some other areas of the body which differ considerably in sensitivity from the fingertips.

If this model of intensity discrimination and masked thresholds is correct, it raises the question of where the interaction between test stimulus and masker is taking place. It is possible that the vibration generated at the site of the masker is physically interacting with the vibration generated at the test site. Measurements of surface waves would indicate that the spread of vibration from the tip of the little finger (masker site) to the tip of 
the index finger (test site) would be small. Using Békésy's (1960) measurements, the amplitude of the surface wave produced by the 20 -micron masker should be 0.0002 microns at the test site. Surface waves are only one indication of the spread of vibration across the body, and therefore it is not possible to rule out physical interaction as an explanation. Several investigators have, however, demonstrated that an intense vibratory stimulus delivered to a site contralateral to a test site will raise the threshold for detection at the test site (Gescheider, Herman, \& Phillips, 1970; Gilson, 1969; Sherrick, 1964). Such results indicate that at least a component of masking must be a central process. If the proposed model were to hold under contralateral masking conditions, i.e., predict the form of the Weber function and masked threshold with several intensity levels of the masker, it would indicate that the addition of the constant proportion of the masker is at a central rather than at a peripheral level.

\section{REFERENCES}

Békésy, G. v. Experiments in hearing. New York: McGraw-Hill, 1960.
Campbell, R. A. Detection of a noise signal of varying duration. Journal of the Acoustical Society of America, 1963, 35, $1732-1735$.

Craig, J. C. Difference threshold for intensity of tactile stimuli. Perception \& Psychophysics, 1972, 11, 150-152.

Gescheider, G. A., Herman, D. D., \& Phillips, J. N. Criterion shifts in the measurement of tactile masking. Perception \& Psychophysics, 1970, 8, 433-436.

Gilson, R. D. Vibrotactile masking: Some spatial and temporal aspects. Perception \& Psychophysics, 1969, 5, 176-180.

Jeffress, L. A. Masking. In J. V. Tobias (Ed.), Foundations of modern auditory theory. Vol. 1. New York: Academic Press, 1971. Pp. 87-114.

Sherrick, C. E. Effects of background noise on the auditory intensive difference limen. Journal of the Acoustical Society of America, 1959, 31, 239-242.

Sherrick, C. E. Effects of double simultaneous stimulation of the skin. American Journal of Psychology, 1964, 77, 42-53.

Talbot, W. H., Darian-Smith, I., Kornhuber, H. H., \& Mountcastle, V. B. The sense of flutter-vibration: Comparison of the human capacity with response patterns of mechanoreceptive afferents from the monkey hand. Journal of Neurophysiology, 1968, 31, 301-334.

(Received for publication May 9, 1973; revision received September 7, 1973.) 